\title{
Coherent learning control of vibrational motion in room temperature molecular gases
}

\author{
T.C. Weinacht ${ }^{\mathrm{a}, *}$, R. Bartels ${ }^{\mathrm{a}}$, S. Backus ${ }^{\mathrm{a}}$, P.H. Bucksbaum ${ }^{\mathrm{b}}$, B. Pearson ${ }^{\mathrm{b}}$, \\ J.M. Geremia ${ }^{c}$, H. Rabitz ${ }^{\text {c}}$, H.C. Kapteyn ${ }^{\mathrm{a}}$, M.M. Murnane ${ }^{\mathrm{a}}$ \\ a JILA, University of Colorado, Boulder, CO 80309-0440, USA \\ b Department of Physics, University of Michigan, Ann Arbor, MI 48109, USA \\ ${ }^{\mathrm{c}}$ Department of Chemistry, Princeton University, Princeton, NJ 08544, USA
}

Received 25 April 2001; in final form 31 May 2001

\begin{abstract}
An evolutionary learning algorithm in conjunction with an ultrafast optical pulse shaper was used to control vibrational motion in molecular gases at room temperature and high pressures. We demonstrate mode suppression and enhancement in sulfur hexafluoride and mode selective excitation in carbon dioxide. Analysis of optimized pulses discovered by the algorithm has allowed for an understanding of the control mechanism. () 2001 Elsevier Science B.V. All rights reserved.
\end{abstract}

Controlling atoms and molecules with coherent optical fields has been a longstanding goal in chemical physics [1]. Recently, several experiments in this field have demonstrated successful control of molecular ionization and dissociation [2,3], atomic and molecular fluorescence [4,5], the shape of electronic and molecular wave packets [6-9], and currents in semiconductors [10,11]. Many of these experiments have employed learning control loops and programmable optical pulse shapers to discover optimal pulse shapes for control [12]. There has been increasing interest in not only achieving control in quantum systems, but also in understanding the control mechanism, which has proven to be a challenging task. Here

\footnotetext{
${ }^{*}$ Corresponding author. Fax: +1-303-492-5235.

E-mail address: weinacht@jilau1.colorado.edu (T.C. Weinacht).
}

we demonstrate two experimental advances in coherent control of quantum systems. First, we demonstrate selective control over molecular motion in gases at STP using very short, shaped excitation pulses. Coherent vibrational excitation corresponding to thermal temperatures over 2000 $\mathrm{K}$ is achieved. This is important in order to reach the goal of laser selective chemistry [13] on a macroscopic scale, because it extends cryogenic and molecular beam experiments to high temperatures and densities. Second, using a special cost function [14] incorporated into an evolutionary learning algorithm [15], we obtain a clear interpretation of the control mechanism, which is based on impulsive stimulated Raman scattering [16]. This serves as a demonstration of a general technique for systematically gaining insight from solutions found by learning algorithms. Furthermore, as there is no molecular resonance exploited in this excitation scheme, and the 
molecules are not specially prepared, the approach is general and can be applied to a large class of molecules.

The two most important time-scales for controlling molecular motion are the vibrational pe$\operatorname{riod}\left(\tau_{\mathrm{v}}\right)$, and the coherence time $\left(\tau_{\mathrm{c}}\right)$. If one is able to generate intense, shaped laser pulses whose duration is shorter than $\tau_{\mathrm{v}}$, then it is possible to manipulate the motion of the molecule on its own natural time-scale. This regime is known as impulsive Raman scattering. Control of molecular motion in the impulsive regime with shaped laser pulses offers many important advantages over steady-state or transient scattering, where the laser pulse is longer than $\tau_{\mathrm{c}}$ and $\tau_{\mathrm{v}}$. One can create and measure vibrational wave packets sensitive to the motion of individual bonds, rather than just populations of eigenstates. Secondly, since the laser pulse is shorter than $\tau_{\mathrm{c}}$, all of the laser energy deposited into the molecules is done so coherently, allowing for efficient molecular excitation (e.g., driving of overtones). Finally, one can programmably alter the phase of a single driving pulse, simultaneously controlling the excitation of several vibrational modes in a given molecule. Attempts to control a comparable number of modes in a molecule with transient or steady-state Raman scattering would require several tunable, picosecond, phaselocked lasers.

The experiment employed intense shaped light pulses with a central frequency of $375 \mathrm{THz}(800$ $\mathrm{nm})$, generated by an ultrashort Ti:Sapphire amplified laser system. The laser system generates temporally shaped pulses as short as $15 \mathrm{fs}$, at a 1 $\mathrm{kHz}$ repetition rate, with over $1 \mathrm{~mJ}$ of energy [17]. A pulse shaper based on a 19 element deformable mirror is inserted between the oscillator and the amplifier [18]. The enormous spectral bandwidth of the pulses produced by the laser system allows for impulsive excitation of room temperature molecules in a relatively pure state, without preselecting a given set of molecules. The FWHM bandwidth of the output laser pulses is over 18 $\mathrm{THz}$, allowing for impulsive excitation of the symmetric breathing mode $\left(v_{1}\right)$ of $\mathrm{SF}_{6}$. At room temperature $(300 \mathrm{~K}), k T=6.24 \mathrm{THz}$, making the thermal population of the first excited level of this mode only 0.024 .
The shaped, amplified pulses from the laser were focussed into a $450 \mu \mathrm{m}$ inner diameter hollow core fiber waveguide, with a length of $30 \mathrm{~cm}$. The waveguide was filled with the molecular gas, with pressures varying from 20 to 1000 Torr. The waveguide could either be filled from a pressurized gas bottle, or by simply connecting a liquid cell to the waveguide and working with the vapor pressure of the molecular liquid. Temporally delayed pulses with a frequency of $750 \mathrm{THz}(400 \mathrm{~nm})$, a bandwidth of $1.5 \mathrm{THz}$, and pulse energy of $1-3 \mu \mathrm{J}$ were injected collinear with the driving pulse in order to probe the vibrational excitation created by the driving pulse. The delay, which was several hundred femtoseconds, was longer than the pump pulse duration but shorter than $\tau_{\mathrm{c}}$. The probepulse spectrum was measured as the shape of the driving pulse was varied. The spectrometer monitoring the probe spectrum was interfaced with a computer, which also controlled the pulse shaper, allowing for closed-loop control of the process. The amplitude and phase of the shaped pulses were measured using second-harmonic frequency resolved optical gating (SHG FROG) ${ }^{1}$ [19-21].

As the probe-pulse propagates through the vibrationally excited gas, it encounters a periodically modulated index of refraction and therefore develops sidebands spaced by the vibrational frequency of the excited modes. As the molecules are prepared in a coherent superposition of $v=1$ and $v=0$, the probe-pulse undergoes both Stokes and anti-Stokes scattering. There is no Stokes sideband from the probe in the absence of the pump, since the scattering of the probe is a result of a coherence between $v=1$ and $v=0$ [22]. The pump pulse also contains signatures of the molecular coherence in the form of a continuous redshift [23] (which is only present for pump pulses that excite vibrations) and a modified temporal profile at higher pressures. This modified temporal structure

\footnotetext{
${ }^{1}$ One potential difficulty with the interpretation of the optimal pulses is reshaping of the pump pulse as a result of self-phase modulation in the waveguide. In order to make a clean interpretation of the optimal pulses found by the algorithm, we made FROG measurements of the pump pulse before and after the waveguide, interpreting our results for gas pressures below which reshaping occurred.
} 
consisted of a pulse train at the vibrational frequency of the mode being excited.

In order to discover optimal pulse shapes for driving and suppressing this molecular coherence, we used an evolutionary algorithm [24] that starts with a collection of population members, each of which corresponds to a particular set of voltages applied to the 19 mirror actuators. The fitness of the each population member is measured experimentally. The fitness is simply a quantitative measure of the vibrational coherence, which in our case, corresponds to the total amount of energy in the probe sidebands for the mode of interest. The best pulse shapes (largest fitness values) are selected as parents for new members of future populations (generations) of the algorithm. Children of the selected parents are generated through mutation using a Gaussian noise function to perturb the solutions. The parents and mutated children are combined to form the population members of the next generation, which are again evaluated and sorted for creating another generation. This process is repeated until the fitness changes by an insignificant amount between generations, at which point the process is said to have converged. This typically occurred in $25-50$ iterations, with about 25 population members per iteration.

In order to learn as much as possible from the solutions discovered by the algorithm, in some cases we incorported a special cost function into the algorithm. The cost function imposes a penalty into the fitness for deviations from an unshaped pulse, limiting unnecessary variations in the optimized pulse shapes. This ensures that all of the differences between an optimized pulse and an unshaped pulse are not merely sufficient, but necessary. The control mechanism is therefore much easier to deduce from the solutions. The cost function, $f_{\text {cost }}$, simply multiplied the experimental fitness, and had the form:

$f_{\text {cost }}=1-\frac{w}{n g_{\max }^{2}} \times \sum_{i=0}^{n}\left(g_{t l}-g_{i}\right)^{2}$.

Here, $w$ is a weighting factor that was adjusted until $f_{\text {cost }}$ was slightly less than $1, g_{\max }$ is the maximum gene value for a given pulse shape, $g_{\mathrm{tl}}$ is the value of each gene for an unshaped pulse, and $g_{i}$ is the gene value for the $i$ th gene for each pulse shape evaluated. Although the values of $g_{t l}$ were set to those of an unshaped pulse in the present experiment, they can be set to bias the cost function toward any pulse shape. Thus the deviations from any given solution can be penalized, in order to discover the essential components of a solution.

Results from exciting and suppressing the totally symmetric breathing mode $\left(v_{1}\right)$ in $\mathrm{SF}_{6}$ at 250 Torr are shown in Fig. 1. Fig. 1a shows the probe spectrum for a pump pulse optimized for mode excitation, while Fig. $1 \mathrm{~b}$ shows the probe spectrum for a pump pulse optimized for mode suppression. Suppression and enhancement is effective over a broad range of pressures (20-700 Torr). We interpret the mechanism in a regime where pulse reshaping during propagation is negligible. The sideband separation of $23.25 \mathrm{THz}$ is in good agreement with the frequency of $v_{1}$ as well as previous measurements [25]. Based on the intensity of the sidebands in the probe spectrum, one can estimate that between $1 \%$ and $10 \%$ of the molecular sample is excited. This result is promising for

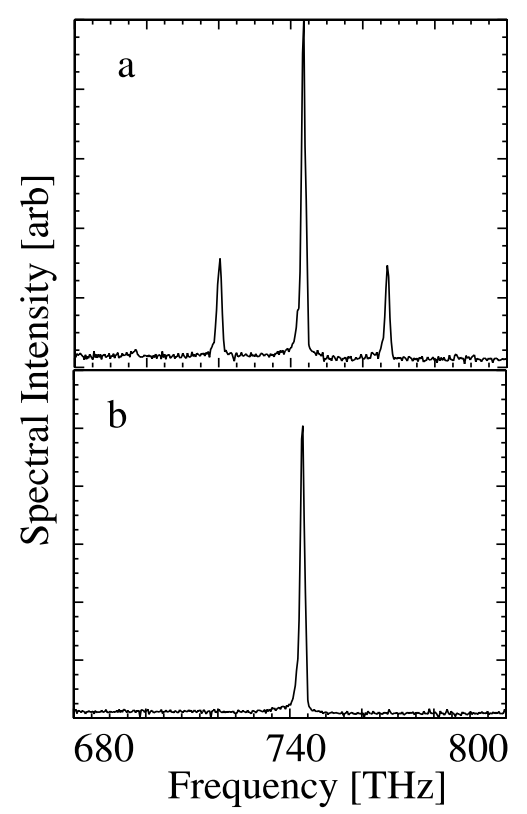

Fig. 1. Probe spectra for pulses optimized to enhance (a) and suppress (b) vibrational excitation in $\mathrm{SF}_{6}$. 
attempting bimolecular chemistry initiated by tailored laser pulses.

There are two strong Raman active modes in $\mathrm{CO}_{2}$ : the asymmetric $\mathrm{CO}$ stretch mode $\left(v_{1}\right)$, and two quanta of the OCO bend mode $\left(v_{2}\right)$. The corresponding Raman shifts are 38.6 and 41.6 $\mathrm{THz}$, respectively. Using the difference in intensity of the sidebands for the two modes as the feedback signal in an evolutionary algorithm, we could make either mode dominant in the excitation. Fig. 2 shows the probe-pulse spectra when the excitation pulse was optimized to drive $v_{1}$ (top panel), or two quanta of $v_{2}$ (bottom panel). These data were taken at a pressure of 280 Torr, with similar results obtained at a pressure of 800 Torr.

The results shown above clearly demonstrate that the excitation of vibrational modes with frequencies less than the laser bandwidth is very sensitive to the shape of the pump pulse. This is in contrast with the steady-state and transient regimes of Raman scattering [26]. It is also in contrast to the usual case of impulsive scattering, in

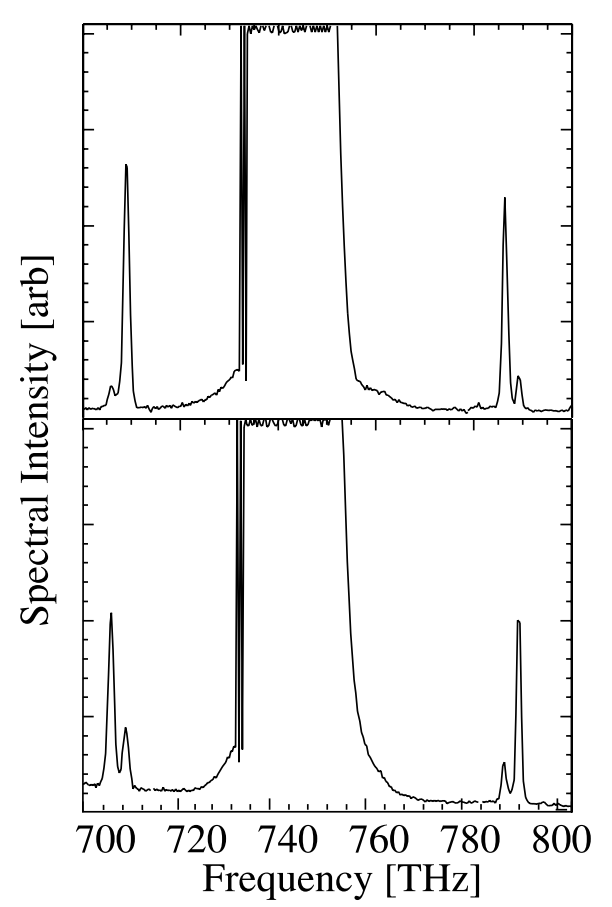

Fig. 2. Probe spectra for pulses optimized to drive $v_{1}$ (top panel) and two quanta of $v_{2}$ in $\mathrm{CO}_{2}$ (bottom panel). which the response is simply proportional to the pulse energy. However, shaping of pulses with enough bandwidth to support impulsive scattering can lead to detailed control over the molecular response [27].

Since the molecule has a significant amount of inertia, it cannot respond instantaneously to the details of the rapidly varying electric field, and the dynamics are insensitive to the absolute temporal phase. What is most relevant for Raman type processes is the phase of frequency differences in the field. For a vibrational mode with a frequency of $\Omega_{\mathrm{v}}$, what matters is the phase of frequency pairs whose difference is equal to $\Omega_{\mathrm{v}}$. In a short pulse with a continuous spectrum, one has to integrate over all frequency pairs separated by $\Omega_{\mathrm{v}}$. If one examines the molecular response in a simple classical model [28] (driven, damped harmonic oscillator), the equation of motion for the atomic displacement, $q$, can be written as

$\frac{\partial^{2} q}{\partial t^{2}}+\gamma \frac{\partial q}{\partial t}+\Omega_{\mathrm{v}}^{2} q=(1 / m)\left[\frac{\partial \alpha}{\partial q}\right]_{0} A_{\mathrm{L}} A_{\mathrm{S}}^{*}$.

Here $m$ is the reduced mass, $\alpha$ is the molecular polarizability, $A_{\mathrm{L}}$ is the complex pump laser field envelope, $A_{\mathrm{S}}$ is the complex Stokes laser field envelope, $\Omega_{\mathrm{v}}$ is the vibrational frequency and $\gamma$ is the damping constant. Transforming both the displacement and driving term to the frequency domain yields

$q(\Omega)=\frac{(1 / m)\left[\frac{\partial \alpha}{\partial q}\right]_{0} \Im\left\{A_{\mathrm{L}} A_{\mathrm{S}}^{*}\right\}}{\Omega_{\mathrm{v}}^{2}-\Omega^{2}-i \Omega \gamma}$.

In the limit of impulsive scattering, the molecular response can be rewritten as

$q(\Omega)=\frac{(1 / m)\left[\frac{\partial \alpha}{\partial q}\right]_{0} \mathfrak{I}\left\{A_{\mathrm{L}} A_{\mathrm{L}}^{*}\right\}}{\Omega_{\mathrm{v}}^{2}-\Omega^{2}-i \Omega \gamma}$.

Since the bandwidth of the pump pulse is larger than the Raman shift, there are frequency components present that can act as a seed for the Stokes pulse and therefore the Stokes wave can be replaced by the laser as shown above. The denominator in Eq. (4) supplies a resonance in the molecular response at $\Omega_{\mathrm{v}}$ so that the molecule is very sensitive to the value of $A_{\mathrm{L}} A_{\mathrm{L}}^{*}$, (the intensity 
envelope, $I(t))$ at $\Omega_{\mathrm{v}}$. Looking at the Fourier transform of $I(t)$ illustrates the connection between $I(t)$ and the phase of frequency differences:

$$
\begin{aligned}
& \left|\mathfrak{J}\left\{A_{\mathrm{L}} A_{\mathrm{L}}^{*}\right\}\right|=\left|\int \mathrm{d} \omega a_{\mathrm{L}}(\omega) a_{\mathrm{L}}^{*}(\Omega-\omega) \mathrm{e}^{\mathrm{i}[\varphi(\omega)-\varphi(\Omega-\omega)]}\right|, \\
& A_{\mathrm{L}}(\omega)=a_{\mathrm{L}}(\omega) \mathrm{e}^{\mathrm{i} \varphi(\omega)} .
\end{aligned}
$$

Fig. 4 shows the intensity envelopes (inset) and their transforms for two pulses optimized to excite a molecular coherence in $\mathrm{SF}_{6}$, and two pulses optimized for suppression. The algorithm found two separate solutions for enhancing and suppressing the vibrations. Each pair of solutions have very different intensity profiles, but nevertheless have nearly the same values in the power spectrum of $I(t)$ at $\Omega_{\mathrm{v}}=23.25 \mathrm{THz}$. The two solutions for optimizing excitation are shown as the green and blue curves in Fig. 3. The red and black curves represent solutions for supressing the vibrational coherence, exhibiting local minima at $\Omega_{\mathrm{v}}$. The green and black curves were taken with the term $f_{\text {cost }}$ included in the algorithm, while the red and blue curves were not so constrained. The cost function in our experiment penalized deviations from an unshaped pulse. The green and black curves show pulses that deviate minimally from an unshaped pulse, while still maintaining control.

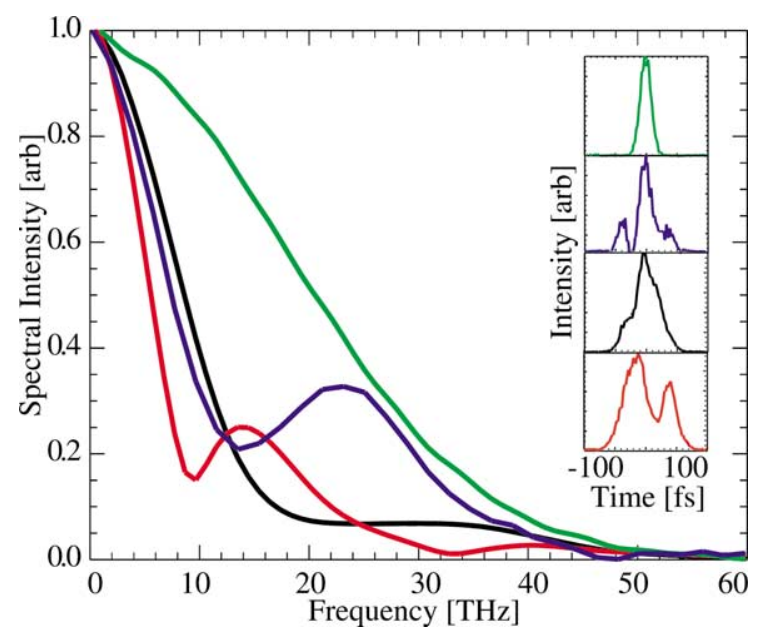

Fig. 3. Intensity profiles and Fourier transforms for pulses optimized to suppress and enhance excitation in $\mathrm{SF}_{6}$.
The differences between the two curves for enhancing the vibrations and the two curves for suppressing the vibrations illustrate the ability of the cost function to help isolate the important features in the optimized pulses - a large or small value in the spectrum of $I(t)$ at $23.25 \mathrm{THz}$ in this case. Comparison of the curves for enhancing and suppressing vibrations supports the impulsive excitation mechanism described above. Analysis of mode selectivity in other molecules (such as $\mathrm{CCl}_{4}$ ) illuminates the same underlying physical mechanism as demonstrated here for $\mathrm{SF}_{6}$ : to enhance or suppress excitation of a given mode, the power spectrum of $I(t)$ at the two photon resonant frequency must be made large or small. This control illustrates how a single broadband laser can be used to control the excitation of $N$ modes in a given molecule, replacing $N+1$ phaselocked narrowband lasers that one would have to tune to the various Raman resonances in order to gain a similar degree of control.

In order to verify the interpretation of the results, we monitored the probe spectrum for several different gases (benzene, carbon tetrachloride, nitrogen and ethylene), and also varied the pump pulse duration without altering the bandwidth. Sidebands spaced by the Stokes shift for a given mode appeared only when the pump pulse duration was less than the vibrational period. As a

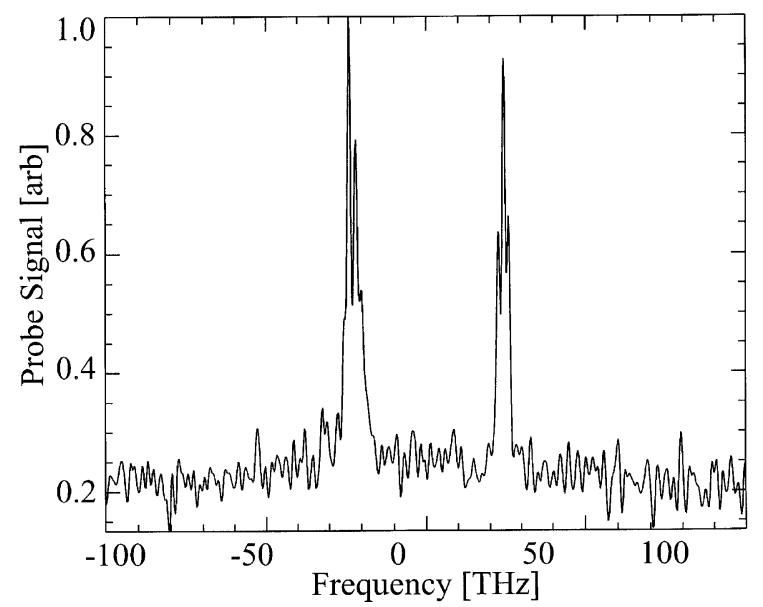

Fig. 4. Probe sideband intensity as a function of frequency difference between two chirped excitation pulses. 
further test, we performed an experiment where we excited the molecules with a chirped pulse pair. Combining the two chirped pulses with a time delay less than their duration yielded a total electric field with a constant frequency difference. The chirp rate, $b$, and the time delay, $\tau$, between the pulses determine the frequency difference, $\omega_{\text {diff }}$, maintained between the two pulses: $\omega_{\text {diff }}=b^{*} \tau$. The value of $b$ was controlled by adjusting the grating separation in the compressor, and measured using SHG FROG. We monitored the sideband intensity in the probe spectrum as a function of $\omega_{\text {diff }}$. Fig. 4 shows the sideband intensity as a function of $\omega_{\text {diff }}$. Experiments with different chirp rates all yielded peaks at $\pm 23( \pm 1)$ $\mathrm{THz}$, in good agreement with the frequency of $v_{1}$ in $\mathrm{SF}_{6}$. Analysis of similar measurements provides strong evidence for overtone excitation [29].

In conclusion, we demonstrate coherent and selective vibrational excitation of molecular gases at room temperatures and high densities. A learning algorithm incorporating a cost function is used to achieve control, yielding a simple interpretation of the control mechanism in terms of impulsive Raman scattering. Significant fractions of the molecular samples were excited. The results are encouraging for the prospect of using tailored laser pulses to provide selective excitations for influencing bimolecular reactions. In the future, since shaped pulses with durations of 5-10 fs are now becoming experimentally practical, it will be possible to extend this approach to all Raman active molecular modes, including $\mathrm{H}_{2}$.

\section{Acknowledgements}

This work was funded by the National Science Foundation. We would like to acknowledge helpful discussions with Paul Corkum and Steve Leone.

\section{References}

[1] H. Rabitz, R. de Vivie-Riedle, M. Motzkus, K. Konpa, Science 288 (2000) 5467, and references therein.
[2] L. Zhu, V. Kleiman, X. Li, S. Lu, K. Trentelman, R.J. Gordon, Science 270 (1995) 77.

[3] A. Assion, T. Baumert, M. Bergt, T. Brixner, B. Keifer, V. Seyfried, M. Strehle, G. Gerber, Science 282 (1998) 919.

[4] C.J. Bardeen, V.V. Yakovlev, K.R. Wilson, S.D. Carpenter, P.M. Weber, W.S. Warren, Chem. Phys. Lett. 280 (1997) 151.

[5] D. Meshulach, Y. Silberberg, Nature 396 (1998) 298.

[6] T.C. Weinacht, J. Ahn, P.H. Bucksbaum, Nature 397 (1998) 233.

[7] M.W. Noel, C.R. Stroud, J. Opt. Express 1 (1997).

[8] T. Hornung, R. Meier, M. Motzkus, Chem. Phys. Lett. 326 (2000) 445.

[9] Z. Amitay, J.B. Ballard, H.U. Stauffer, S.R. Leone, Chem. Phys. 267 (2001) 69.

[10] A. Hache, Y. Kostoulas, R. Atanasov, J.L.P. Hughes, J.E. Sipe, H.M. van Driel, Phys. Rev. Lett. 78 (1997) 306.

[11] E. Dupont, P.B. Corkum, H.C. Liu, M. Buchanan, Z.R. Wasilewski, Phys. Rev. Lett. 74 (1995) 3596.

[12] R. Judson, H. Rabitz, Phys. Rev. Lett. 68 (1992) 1500.

[13] R.N. Zare, Science 279 (1998) 1875.

[14] J.M. Geremia, W.J. Zhu, H. Rabitz, J. Chem. Phys. 113 (2000) 10841.

[15] H.P. Schwefel, Evolution and Optimum Seeking, Wiley, New York, 1995.

[16] A.M. Weiner, D.E. Leird, G.P. Wiederrecht, K.A. Nel, Science 247 (1990) 1317.

[17] E. Zeek, R. Bartels, M.M. Murnane, H.C. Kapteyn, S. Backus, G. Vdovin, Opt. Lett. 25 (2000) 587.

[18] G. Vdovin, S. Middlekoch, P.M. Sarro, Opt. Eng. 36 (1997) 1382.

[19] K.W. DeLong, R. Trebino, D.J. Kane, JOSA B 11 (1994) 1595.

[20] K.W. DeLong, R. Trebino, J. Hunter, W.E. White, JOSA B 11 (1994) 2206.

[21] D.J. Kane, R. Trebino, IEEE J. Quantum Electron. 29 (1993) 571.

[22] M. Wittmann, A. Nzarkin, G. Korn, Phys. Rev. Lett. 84 (2000) 5508.

[23] G. Korn, O. Duhr, A. Nazarkin, Phys. Rev. Lett. 81 (1998) 1215.

[24] H.P. Schwefel, G. Rudolph, Adv. Artif. Life 929 (1995) 893.

[25] H.W. Schrotter, H.W. Klockner, in: A. Weber, Raman Scattering, Springer, Berlin, 1970.

[26] R.L. Carmen, F. Shimizu, C.S. Wang, N. Bloembergen, Phys. Rev. Lett. 2 (1979) 60.

[27] D. Meshulach, Y. Silberberg, Phys. Rev. A. 60 (1999) 1287.

[28] R. Boyd, Nonlinear Optics, Academic Press, New York, 1992.

[29] R. Bartels, T.C. Weinacht, S.R. Leone, H.C. Kapteyn, M.M. Murnane, Phys. Rev. Lett. (submitted). 